\title{
LES VARIATIONS PONDÉRALES DU TISSU ADIPEUX ET DES OVAIRES, ET LES VARIATIONS DE LONGUEUR DES OVOCYTES, CHEZ IPS SEXDENTATUS BOERN (COLEOPTERA : SCOLYTIDAE); RELATIONS AVEC LE PARASITISME PAR LES NÉmATOdeS
}

\author{
F. LIEUTIER* \\ Collaboration technique : Mmes M. Jastrabsky et P. Bonnafe
}

RÉSUMÉ. Les variations du poids du tissu adipeux et des ovaires, ainsi que la longueur de l'ovocyte le plus mûr, ont été étudiées chez Ips sexdentatus, depuis la fin de la maturation de préessaimage, jusqu'à la $24^{\mathrm{e}}$ heure après l'accouplement.

Chez les Insectes non parasités, le poids des ovaires et la taille de l'ovocyte terminal s'accroissent jusqu'à l'essaimage, mais régressent légèrement à la $3^{\mathrm{e}}$ heure après l'accouplement ; la croissance reprend ensuite, d'abord lente, puis très rapide après la $12^{\mathrm{e}}$ heure. Le tissu adipeux régresse au moment de l'essaimage, mais s'accroît nettement à la $6^{\mathrm{e}}$ heure ; son poids diminue ensuite fortement à la $12^{\mathrm{e}}$ et $18^{\mathrm{e}}$ heure, puis croît à nouveau de façon importante à la $24^{\mathrm{e}}$ heure. Les variations du poids des ovaires (et de la taille de l'ovocyte terminal) peuvent être reliées à celles du poids du tissu adipeux, si l'on tient compte de l'existence d'un certain retard dans la " réponse » des ovaires. L'ensemble de ces variations serait le reflet de phases importantes de la biologie du scolytide: essaimage, maturation lente puis rapide des ovaires, ponte.

Chez les insectes parasités par les Nématodes Parasitaphelenchus sp., P. sexdentati (Fuchs 1937) Rühm 1956, Contortylenchus diplogaster (V. Lins 1890) Rühm 1956, le tissu adipeux et les ovaires sont toujours plus légers, et l'ovocyte terminal toujours moins développé, que chez les Insectes indemnes.

De plus, le tissu adipeux des Insectes contaminés ne peut constituer les réserves nécessaires à la maturation des ovaires aux moments où ceux-ci semblent en avoir le plus besoin. Il s'ensuit que la maturation des ovocytes de ces Insectes est retardée. Ce retard, se cumulant pour les ovocytes successifs, pourrait expliquer la diminution importante de la ponte chez les Insectes parasités.

L'action des Nématodes sur les ovaires d'Ips sexdentatus serait donc indirecte, l'action directe pouvant s'exercer alors sur le tissu adipeux ou l'hémolymphe. Il semble d'autre part que le tissu adipeux abdominal soit plus affecté par le parasitisme que le tissu adipeux prothoracique.

The variations of the weight of the fat body and the ovaries, and the variations of the length of the ovocytes in Ips sexdentatus (Coleoptera : Scolytidae); relations with the parasitism by the nematodes.

SUMMARY. The variations of the weight of the fat body and the ovaries, as well as the length of the maturest oocyte, have been studied in Ips sexdentatus, from the end of the maturation of pre-swarming to the 24 th hour after the copulation.

* Laboratoire de Zoologie, Institut National Agronomique, 16, rue Claude Bernard, F 75005 Paris Accepté le 3 février 1982 
In the healthy Insects, the weight of the ovaries and the size of the terminal oocyte grow till the swarming, but decline slightly at the 3rd hour after the copulation; the growth recovers afterwards, at first slow, then very steep after the 12th hour. The fat body declines at the swarming, but grows distinctly at the 6 th hour; its weight decreases strongly at the 12 th and 18 th hour, then grows again considerably at the $24 \mathrm{th}$ hour. The variations of the weight of the ovaries (and of the size of the terminal oocyte) may be compared with that of the fat body, if we take into consideration the being of a delay in the " response $»$ of the ovaries. This combined variations might be agreed with important periods of the biology of the bark-beetle : swarming, slow then steep maturation of the ovaries, oviposition.

In the Insects parasitized by the Nematodes Parasitaphelenchus sp., P. sexdentati (Fuchs 1937) Rühm 1956, Contortylenchus diplogaster (V. Lins 1890) Rühm 1956, the fat body and the ovaries are always slighter, and the terminal oocyte less developped than in the healthy Insects.

Moreover, the fat body of the infected Insects cannot accumulate the indispensable stocks for the maturation of the oocytes, just as these one seem to want that. Therefore the maturation of the oocytes of this Insects is delayed. This delay, by accumulating itself for the successive oocytes, might explain the important reduction of the oviposition in the parasitized Insects.

The effect of the Nematodes on the ovaries of I ps sexdentatus might be indirect, the direct effect being related to the fat body or to the blood. On the otherside, the abdominal fat body seems to be more affected by the parasitism than the prothoracic one.

Les Nématodes endoparasites sont la cause, chez Ips sexdentatus Boern., d'un certain nombre de perturbations qui entraînent en particulier un retard dans la maturation de l'insecte. Ce retard se traduit par un essaimage et une ponte plus tardifs chez les insectes parasités (Lieutier 1981, 1982). De plus la fécondité de ces insectes est fortement réduite (Lieutier 1982).

Dans le but d'examiner à quels processus physiologiques correspondent ces modifications comportementales, nous avons entrepris une étude du tissu adipeux, des ovaires et de l'hémolymphe d'I ps sexdentatus. Dans un premier temps, nous présentons les résultats de nos investigations à un niveau global, concernant l'importance du tissu adipeux et des ovaires.

L'action des Nématodes Tylenchida sur les ovaires ou le tissu adipeux des Scolytidae a été rapportée par un certain nombre d'auteurs.

Chez les Ipinae, Fuchs (1915) et Rühm (1956) indiquent une influence de Parasitylenchus dispar (Fuchs 1915) Filipjev 1934 et de C. diplogaster sur Ips typographus L. Rühm (1956) signale également l'action de Parasitaphelenchus papillatus Fuchs 1937 sur Blastophagus minor Hart. et B. piniperda L., ainsi que celle des Neoparasitylenchus et des Parasitylenchus sur divers autres Scolytidae; et Gurando (1974) celle de Parasitaphelenchus sp. et de Parasitylenchus macrobursatus Blinova et Gurando 1977 sur B. minor Hart. Les résultats de Laumond et Carle (1971) semblent indiquer aussi une action de $P$. papillatus, associé à Parasitorhabditis piniperdae (Fuchs 1937) Rühm 1956, sur le tissu adipeux et les ovaires de Blastophagus destruens Woll. Sulphuretylenchus sp. réduirait la taille des gonades d'Ips confusus Lec. (Nickle 1963). Thong et Webster (1975) signalent une diminution de $20 \%$ de la taille des ovocytes de Dendroctomus pseudotsugae Hopk. infesté par Contortylenchus reversus (Thorne 1935) Rühm 1956. 
Dans la sous-famille des Scolytinae, chez Scolytus multistriatus Marsh. et S. ssolytus Fab., les ovaires des insectes parasités par Neoparasitylenchus scolyti (Oldham 1930) Nickle 1967 et Parasitaphelenchus oldhami Rühm 1956 sont souvent atrophiés (Oldham 1930, Chararas 1957, 1962). Il en est de même de ceux de Ruguloscolytus rugulosus Ratz. parasité par Neoparasitylenchus rugulosi (Schvester 1957) Nickle 1967, (Schvester 1957), et de ceux de Scolytus ventralis Lec., parasité par Sulphuretylenchus elongatus (Massey 1958) Nickle 1967, (Massey 1964, Ashraf et Berryman, 1970 a, b). Dans ce dernier cas, le tissu adipeux est également modifié.

Néanmoins, Saunders et Norris (1961) chez S. multistriatus et Hoffard et Coster (1976) chez Ips grandicollis Eichh. et Ips avulsus Eichh. n'ont pas noté d'action des Nématodes parasites sur les ovaires.

L'action des Tylenchida sur les organes internes d'autres Insectes que les Scolytidae a été également signalée dans de nombreux cas (Bovien 1937, Palm 1948, Wachek 1955, Pouvreau 1962, entre autres).

Si donc l'action de ces Nématodes sur les ovaires et le tissu adipeux des Insectes et en particulier des Scolytides, a fait l'objet de nombreuses études, aucune de cellesci n'a cependant concerné $I$. sexdentatus dont l'importance économique dans les forêts de Pins est certaine en Europe.

Trois types de Nématodes parasitent la cavité générale d'I $p$ s sexdentatus, ce sont : Contortylenchus diplogaster (V. Lins. 1890) Rühm 1956 (Allantonematidae), Parasitaphelenchus sexdentati (Fuchs 1937) Rühm 1956 et Parasitaphelenchus sp. (Aphelenchoïdidae). La biologie du Scolyte, les cycles des Nématodes, ainsi que la localisation de ceux-ci dans leur hôte ont déja été décrits (Lieutier, 1979, 1980, Lieutier et Seureau, 1981). Nous rappellerons simplement ici que chez les Parasitaphelenchus, ce sont les larves qui contaminent les Insectes, et que ces Nématodes sont considérés comme des parasites facultatifs (Poinar 1972, 1975). Par contre, ce sont les femelles, les œufs et les larves de Contortylenchus qui sont trouvés dans les Insectes, et ce Nématode, comme tous les Allantonematidae, est considéré comme un parasite obligatoire (Poinar 1975).

\section{Matériel et méthodes}

- Étude des stades de maturation des adultes avant accouplement (mâles et femelles) :

Elle concerne les Insectes en fin de maturation (de couleur brun foncé ou noire) et les Insectes en essaimage. Les premiers ont été récoltés sur le terrain et classés en deux catégories selon qu'ils étaient encore en cours d'alimentation (intestin moyen rempli d'aliments), ou que celle-ci était terminée (intestin moyen vide). Des écorces de Pin sylvestre infestées par des adultes en fin de maturation ont été installées au laboratoire en cage d'élevage ; les insectes essaimant ont alors été récoltés au fur et à mesure de leurs sorties.

- Étude sur les femelles en maturation de ponte :

Elle a eu lieu au laboratoire ; le dispositif expérimental a été décrit précedemment (Lieutier, 1982). Il permet de récolter les femelles d'I $P s$ à des temps variés après 
l'accouplement (3 heures, 6 h., 12 h., 18 h., 24 h.), de façon à suivre leur évolution au cours de la maturation de ponte.

- Dans tous les cas, les Insectes récoltés étaient disséqués dans la solution de Ringer et le parasitisme noté.

Pour chaque Insecte, le tissu adipeux récupéré était placé sur une petite plaquette de verre préalablement tarée, et le Ringer entraîné sur la plaquette par le tissu adipeux était ensuite pompé au moyen de tubes capillaires. Par pesée, nous obtenions alors le poids frais de tissu adipeux. Pour un certain nombre d'Insectes en fin de maturation de préessaimage, et pour la quasi-totalité des Insectes en maturation de ponte, des pesées séparées ont été réalisées pour le tissu adipeux se trouvant au niveau du prothorax d'une part (tissu adipeux prothoracique) et celui se trouvant dans l'abdomen et les deux derniers segments thoraciques d'autre part (tissu adipeux " abdominal »).

Les ovaires étaient prélevés et pesés de la même manière que le tissu adipeux, après avoir mesuré la longueur de l'ovocyte le plus mûr.

\section{Résultats}

\section{1 - Le tissu adipeux ( fig. 1)}

Pour la quasi-totalité des types de parasites et de stades de développement de l'Insecte, le poids du tissu adipeux des Insectes parasités est nettement inférieur à celui des non parasités.

Chez les femelles en maturation avant l'accouplement, la différence est significative, pour les deux types de parasites, à partir du moment où les Insectes ont terminé leur alimentation. Chez les femelles en maturation de ponte, parasitées par $P$. sexdentati ou Parasitaphelenchus sp. la différence devient significative à partir de la $6^{\mathrm{e}}$ heure après l'accouplement. Nous disposons de très peu de résultats, pour cette phase de maturation, quant au parasitisme par $C$. diplogaster : à la $24 \mathrm{e}$ heure, le tissu adipeux des femelles contaminées par ce Nématode est moins important que celui des femelles non parasitées, mais la différence n'est toutefois pas significative au niveau $95 \%$.

Chez les Insectes mâles, bien que le tissu adipeux soit presque toujours nettement moins lourd chez les Insectes parasités que chez les non parasités, la différence n'est jamais significative au niveau $95 \%$.

Nous pouvons remarquer également que dans tous les cas (mâles ou femelles, parasités ou non), le poids du tissu adipeux diminue de façon importante chez les insectes en essaimage, alors qu'il est sensiblement le même lors des deux stades de maturation précédents. Plus tard, chez les femelles en maturation de ponte, qu'elles soient ou non parasitées, le poids du tissu adipeux atteint sa valeur maximum à la $6^{\mathrm{e}}$ heure après l'accouplement, puis diminue rapidement. Il remonte ensuite nettement à la $24^{\mathrm{e}}$ heure chez les Insectes non parasités, alors qu'il se maintient à une faible valeur chez les parasités.

D'autre part, chez les Ips non infestés, les valeurs sont comparables entre les mâles et les femelles de même stade, alors que chez les $I p s$ infestés, les femelles ont un tissu adipeux légèrement moins lourd que celui des mâles. 


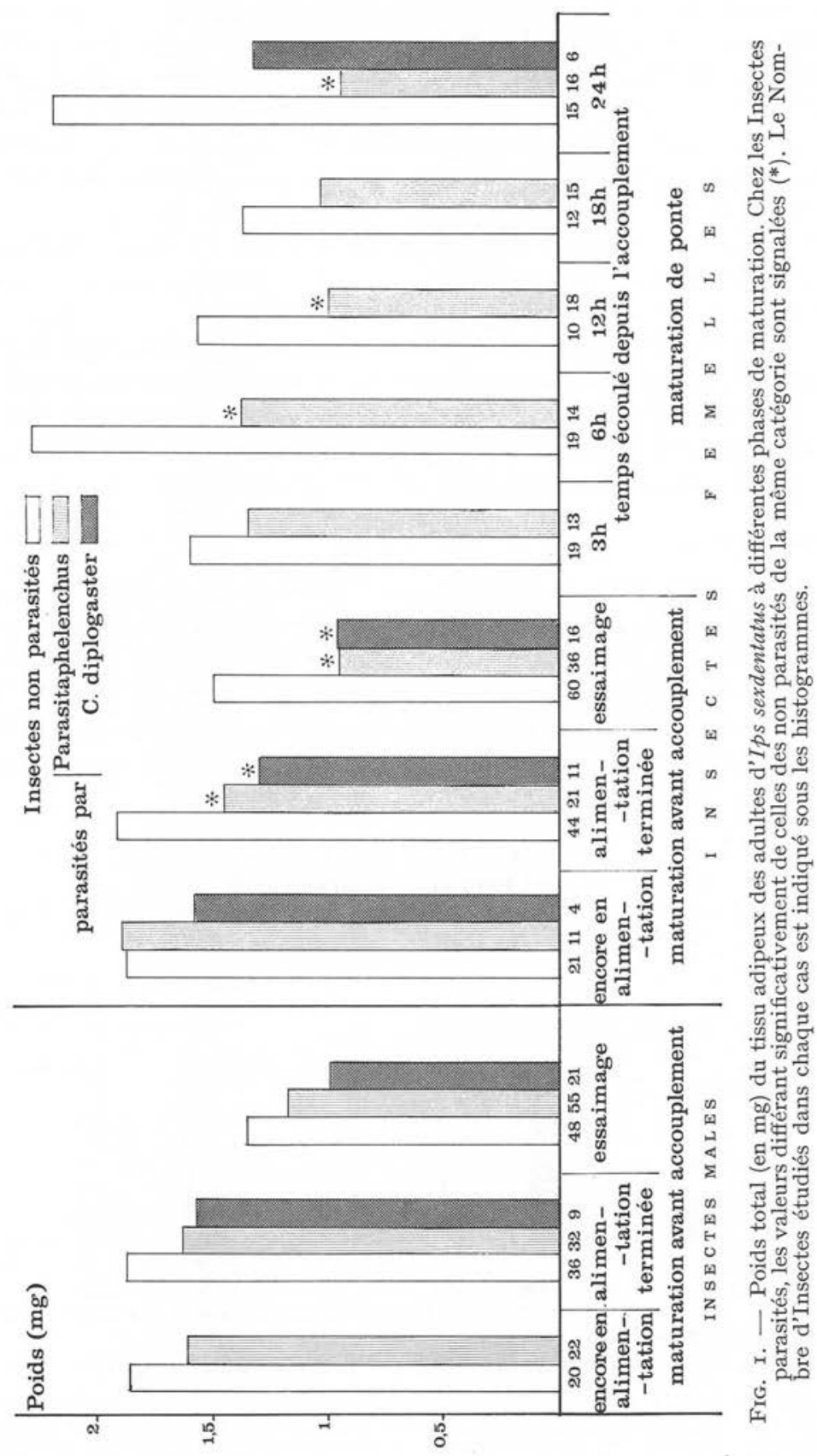


La mesure de l'importance (\%) du tissu adipeux " abdominal " par rapport au tissu adipeux total, en fonction des divers stades de maturation des femelles d' $I p s$, nous a permis de constater que dans tous les cas, chez les Insectes n'ayant pas terminé leur alimentation, le tissu adipeux " abdominal » représente de 55 à $66 \%$ du tissu adipeux total. Il diminue par la suite, surtout chez les femelles en maturation de ponte. De plus, pour celles qui ne sont pas parasitées, il représente de 52 à $54 \%$ du total (exception $46 \%$ à la $18^{\mathrm{e}}$ heure après l'accouplement), alors qu'il est constamment inférieur à $50 \%$ chez les Insectes contaminés par les Parasitaphelenchus (45 à $49 \%$ ). Le faible nombre de résultats concernant le parasitisme par $C$. diplogaster va dans le même sens ( $47 \%$ à la $24 \mathrm{e}$ heure).

Les tests d'homogénéité que nous avons pu effectuer d'autre part sur les quantités de tissu adipeux, nous ont néanmoins montré que, lorsque les différences entre Insectes parasités et non parasités en maturation de ponte étaient significatives pour le tissu adipeux total, elles l'étaient aussi dans la plupart des cas pour le tissu adipeux "abdominal " en même temps que pour le tissu adipeux prothoracique.

\section{2 - Les ovaires (fig. 2)}

Les ovaires des Insectes parasités par les Parasitaphelenchus sont toujours plus légers que ceux des non parasités, surtout à partir du moment où l'alimentation avant essaimage est terminée. La différence n'est cependant significative qu'au moment de l'essaimage, et à la $18 \mathrm{e}$ heure après l'accouplement. Les ovaires des femelles parasités par $C$. diplogaster sont aussi plus légers que ceux des non parasitées, mais la différence n'est pas significative au niveau $95 \%$.

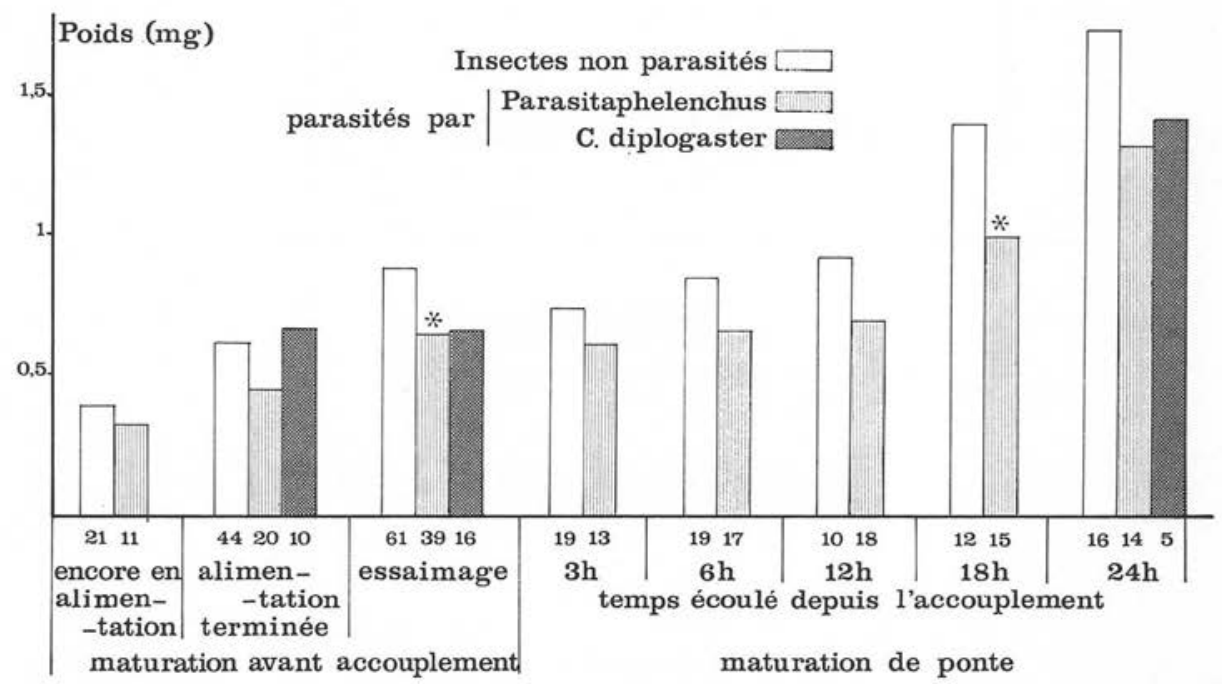

FIG. 2. - Poids (en mg) des ovaires d'Ips sexdentatus à différentes phases de maturation de l'Insecte. Chez les Insectes parasités, les valeurs différant significativement de celles des non parasités sont signalées $\left(^{*}\right)$. Le nombre d'Insectes étudiés dans chaque cas est indiqué sous les histogrammes. 
D'autre part, chez les Insectes parasités comme chez les non parasités, le poids des ovaires augmente au cours de la maturation avant essaimage ainsi qu'au cours de la maturation de ponte. Celle-ci débute néanmoins ( $3^{\mathrm{e}}$ heure après l'accouplement) à une valeur légèrement inférieure à celle de l'essaimage. La croissance pondérale des ovaires s'accélère nettement après la $12^{\mathrm{e}}$ heure.

La mesure de la longueur de l'ovocyte le plus mûr (fig. 3) fournit des résultats analogues : la taille de cet ovocyte, chez les Insectes parasités, est constamment inférieure à celle mesurée chez les non parasités, mais la différence n'est significative que quand l'alimentation avant essaimage est terminée, et à la $18^{\mathrm{e}}$ heure après l'accouplement. Aucune différence n'est cependant notée à la 24e heure.

La longueur de l'ovocyte terminal croît jusqu'à l'essaimage ; après une légère diminution à la $3^{e}$ heure après l'accouplement, elle s'accroît à nouveau au cours de la maturation de ponte, d'abord lentement, puis très rapidement après la $12^{\mathrm{e}}$ heure.

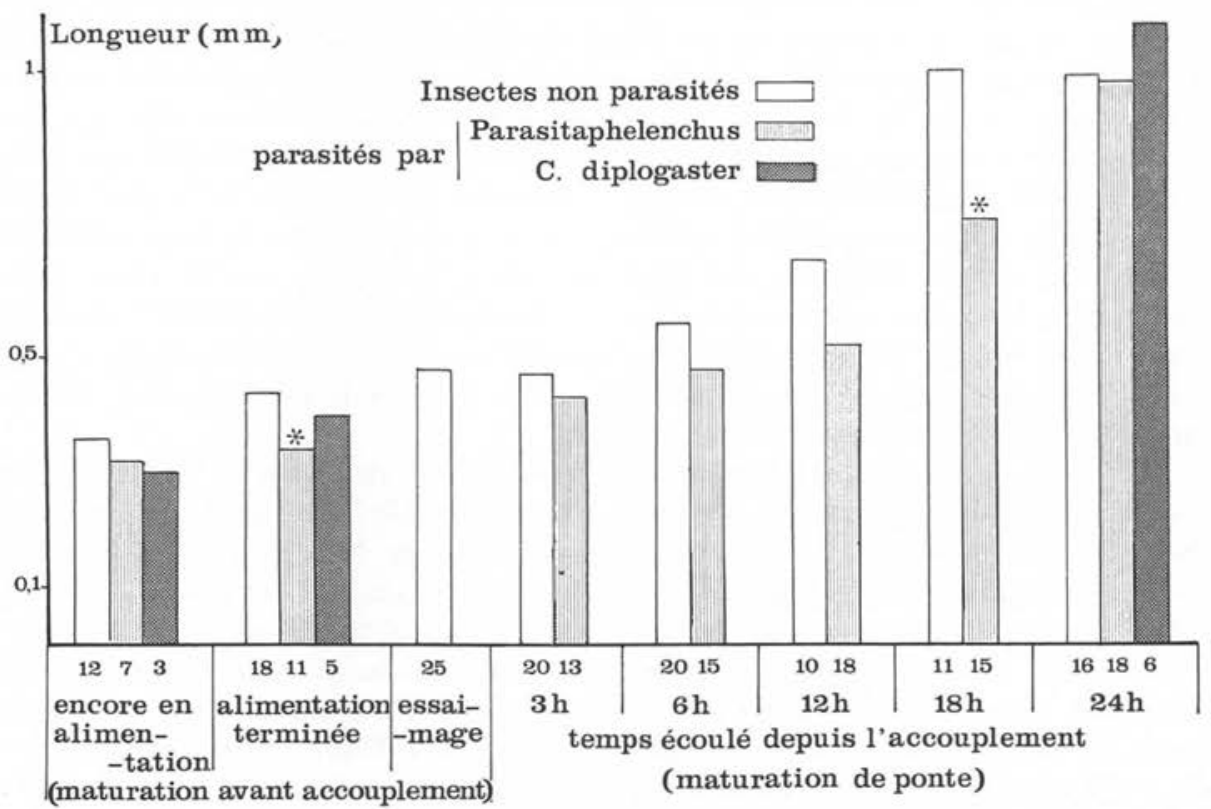

FIG. 3. - Longueur (en mm) de l'ovocyte le plus mûr, chez Ips sexdentatus à différentes phases de maturation. Chez les Insectes parasités, les valeurs différant significativement de celles des non parasités sont signalées $\left(^{*}\right)$. Le nombre d'Insectes étudiés dans chaque cas est indiqué sous les histogrammes.

\section{Interprétations et discussion}

Le parasitisme par les Parasitaphelenchus et par $C$. diplogaster a donc une action certaine sur le tissu adipeux et les ovaires des femelles d'I $p s$ sexdentatus.

Dans quelques cas, les différences entre Insectes parasités et non parasités ne sont pas significatives. Cependant, elles ont lieu dans ces cas toujours dans le même 
sens ; nous pensons donc qu'elles ne peuvent être le fait du seul hasard, et qu'elles correspondent à une réelle influence des parasites sur les Insectes.

L'interprétation de nos résultats est essentiellement basée sur l'action des Parasitaphelenchus ; mais les résultats dont nous disposons pour $C$. diplogaster sont similaires, et n'interdisent donc pas de penser que l'action de ce Nématode est comparable à celle des Parasitaphelenchus, ainsi que nous avons déjà été amené à le signaler (Lieutier, 1982).

$\mathrm{Au}$ cours de la phase de maturation avant essaimage que nous avons étudiée, l'action des parasites sur le tissu adipeux ne devient sensible que lorsque l'alimentation des Insectes est terminée. Au cours de la période d'alimentation, les réserves de l'Insecte utilisées par les Nématodes sont en effet constamment reconstituées, ce qui n'est pas le cas quand l'alimentation a cessé. Il est donc normal que l'action des parasites ne soit visible qu'à cette période sur le tissu adipeux.

Le poids des ovaires et la taille de l'ovocyte le plus mûr augmentent assez régulièrement jusqu'à l'essaimage, tandis que le poids du tissu adipeux diminue pendant le même temps. Ce phénomène existe aussi bien chez les $I p s$ parasités que chez les non parasités. Il est sans doute la conséquence du fait que la maturation des ovocytes et des ovaires s'effectue en partie aux dépens des réserves du tissu adipeux, par l'intermédiaire de l'hémolymphe. Il correspond aussi certainement à l'utilisation d'une partie de ces réserves pendant le début du vol d'essaimage dans la cage d'élevage, puisque l'on observe également une diminution du tissu adipeux chez les mâles. Chez les Insectes parasités, cette diminution est néanmoins plus importante chez les femelles que chez les mâles, ce qui semble indiquer que l'action des Nématodes s'ajouterait à celle des ovaires et du vol pour épuiser les réserves du tissu adipeux, directement ou par l'intermédiaire de l'hémolymphe.

Après l'accouplement et le début de l'alimentation des femelles (3e heure), les réserves du tissu adipeux se reconstituent, comme semble l'indiquer l'augmentation du poids de celui-ci par rapport à la période d'essaimage. Néanmoins, les ovaires et l'ovocyte terminal semblent montrer une légère régression à ce moment. Ce phénomène, apparemment curieux, peut s'expliquer dans l'hypothèse d'une action indirecte du vol, et des Nématodes pour les Insectes parasités, sur les ovaires ; l'action directe s'exerçant alors sur le tissu adipeux ou l'hémolymphe. Un certain délai serait nécessaire à la "réponse " des ovaires, et leur régression à la $3^{\mathrm{e}}$ heure serait la conséquence du vol d'essaimage. Cette hypothèse suppose également que lorsque la "demande » est très forte de la part de l'activité de vol, et de la part des Nématodes pour les femelles parasitées, les ovaires peuvent restituer une partie des substances qu'ils ont puisées dans l'hémolymphe. Ce phénomène de régression ovocytaire sous l'effet des Nématodes parasites a déjà été signalé chez d'autres Insectes, en particulier chez Schistocerca gregaria Forskäl. parasité par Mermis nigrescens Dujardin (Gordon et coll., 1973), et chez les reines de bourdons parasitées par Sphaerularia bombi Dufour (Pouvreau, 1962).

De la $3^{\mathrm{e}}$ à la $6^{\mathrm{e}}$ heure, la croissance des ovaires et de l'ovocyte terminal est lente ; ce qui permet, chez les Insectes indemnes, la constitution de réserves importantes dans le tissu adipeux. Après la $12^{\mathrm{e}}$ heure, la maturation de l'ovocyte s'accélère de 
façon très importante. Une observation analogue chez les Scolytidae a été rapportée par Sahota et coll., (1970) chez Dendroctonus pseudotsugae Hopk. : la majeure partie de l'accroissement des ovocytes de cet Insecte a lieu en effet après la $48^{\mathrm{e}}$ heure suivant l'installation des femelles sur les arbres, alors que la ponte débute à la $96^{\mathrm{e}}$ heure. Sahota et Ibaraki (1973) ont de plus montré que le dépôt des protéïnes de vitellogénèse dans les ovocytes du même Insecte n'avait pas lieu non plus avant la $48^{\mathrm{e}}$ heure. Dans notre étude, il est possible que cette accélération soit due au moins en partie à l'existence dans le lot étudié, d'Insectes à ponte précoce, puisque celle-ci peut débuter dès la $18^{\mathrm{e}}$ heure chez les $I p s$ non parasités (Lieutier, 1982). Néanmoins, quelque soit le cas, la mobilisation au préalable d'une grande partie des réserves du tissu adipeux a été nécessaire, ce qui explique, compte tenu du délai invoqué précedemment, la chute importante du poids de celui-ci dès la $12^{\mathrm{e}}$ heure chez les Insectes non parasités. Sahota (1970) a d'ailleurs montré chez D. pseudotsugae Hopk. que l'apparition des protéines de vitellogénèse dans les ovaires avait lieu nettement plus tard que dans l'hémolymphe.

Chez les Insectes parasités, la maturation ovarienne, très lente de la $3^{\mathrm{e}}$ heure à la $12^{\mathrm{e}}$ heure, s'accélère également après la $12^{\mathrm{e}}$ heure. Mais la présence des Nématodes a empêché la constitution de réserves importantes dans le tissu adipeux à la $6^{\mathrm{e}}$ heure ; la mobilisation des réserves est alors difficile à la $12^{\mathrm{e}}$ heure : le tissu adipeux atteint à ce moment un poids très faible, et les ovaires n'ont pas à leur disposition tout ce qu'il leur faudrait. Leur retard de maturation s'accentue alors.

A la $24 \mathrm{e}$ heure, la majorité des Insectes non infestés s'apprête ou a déjà commencé à pondre (Lieutier, 1982); certains ont même pondu deux ou parfois trois œufs. D'autre part, nous avons pu observer par dissection que la maturation du $2^{\mathrm{e}}$ et du $3^{\mathrm{e}}$ ovocyte était souvent déjà assez avancée au moment de la première ponte. Ces faits expliquent les valeurs élevées du poids des ovaires et de la longueur de l'ovocyte terminal.

Chez les Insectes parasités, le poids des ovaires et la taille de l'ovocyte terminal, à la $24^{\mathrm{e}}$ heure, ont a peu près la même valeur que chez les Insectes indemnes à la $18^{e}$ heure, traduisant ainsi une partie du retard. La ponte débutera donc pour ces Insectes plus tard que pour les non parasités. Nous avons d'ailleurs constaté que 3 jours après l'accouplement, $33 \%$ des Insectes parasités n'ont pas encore pondu (Lieutier, 1982). Par suite, la maturation du $2^{\mathrm{e}}$ ovocyte s'effectuera également plus tard, et si les Nématodes ne permettent toujours pas, comme à la $6^{\mathrm{e}}$ heure, la constitution des réserves dans le tissu adipeux, cette maturation sera aussi plus lente. Ainsi, les retards s'accumulent de proche en proche, et peuvent être la cause du moins grand nombre d'œufs pondus par les Insectes parasités.

L'hypothèse d'une action indirecte des Nématodes sur les ovaires d'Ips sexdentatus permet donc de rendre compte des résultats observés ici, et d'expliquer les modifications de la ponte constatées précédemment (Lieutier, 1982). Cette action s'exercerait par l'intermédiaire du tissu adipeux, lequel chez les Insectes est à l'origine d'une grande partie des substances nécessaires à la croissance ovarienne.

C'est l'ensemble du tissu adipeux de l'hôte qui serait affecté par la présence des parasites, mais il est néanmoins possible que le tissu abdominal en souffre plus que le 
tissu adipeux prothoracique. Ce fait serait alors à rapprocher de la localisation préférentielle des Nématodes dans l'abdomen de l'Insecte (Lieutier 1979, 1980), mais aussi certainement de la localisation des ovaires, et en particulier de l'ovocyte terminal, dans cette même zone.

Au niveau du tissu adipeux, l'action des Nématodes peut être directe, s'ils se développent au contact de cet organe, ou indirecte si l'on suppose que, les parasites puisant dans l'hémolymphe les substances qui leur sont nécessaires, la régulation de la concentration sanguine provoque la " fonte " du tissu adipeux. Or, le mode d'alimentation des Nématodes parasites dans les Insectes est probablement transcuticulaire. C'est certainement le cas chez les Mermithides, puisque Poinar et Hess (1977) ont observé des pores pouvant servir au passage des nutriments, dans la cuticule des larves de Romanomermis culticivorax parasites de Culex pipiens L. Chez les Allantonematidae, des structures favorisant une absorption transcuticulaire semblent également exister chez les Howardula (Riding, 1970) et les Heterotylenchus (Nicholas, 1972), et il n'est pas impossible qu'il en soit de même chez les Contortylenchus, genre voisin des Heterotylenchus (Thong et Webster, 1975). On ne sait malheureusement rien à ce sujet chez les Parasitaphelenchus. D'autre part, les molécules absorbées par les parasites sont de très petite taille. C'est ainsi que les Mermithides puiseraient dans l'hémolymphe de leurs hôtes essentiellement des acides aminés et des sucres simples tels que le glucose; les protéines, les peptides, et le tréhalose n'étant pas absorbé (Gordon et Webster, 1972, Rutherford et coll., 1977, Rutherford et Webster, 1978).

\section{En conséquence :}

- Dans l'hypothèse d'une action directe des Nématodes sur le tissu adipeux de leur hôte, il faut faire intervenir une lyse importante de ce tissu, sous l'action par exemple de sécrétions enzymatiques du Nématode, comme le suppose Rubtsov (1967) chez les Simulies parasitées par les Mermithides. Ce mode d'action directe est à notre avis possible pour $C$. diplogaster, puisque ce Nématode peut se localiser, pendant au moins une partie de son développement parasitaire, à l'intérieur du tissu adipeux de son hôte, dans un syncytium dont il a induit la formation à partir de ce tissu (Lieutier et Seureau, 1981).

- Cela n'exclue pas la possibilité d'une action indirecte sur le tissu adipeux, en particulier pour les Parasitaphelenchus et même pour Contortylenchus. Une telle possibilité à été envisagée pour divers Mermithides parasites de Criquets ou de Simulies. Ces nématodes modifieraient le métabolisme de leurs hôtes (par stimulation du catabolisme - ou diminution de l'anabolisme - des protéines et du glycogène), et favoriseraient ainsi la synthèse dans l'hémolymphe de petites molécules, acides aminés et glucose en particulier, à partir des réserves du tissu adipeux. Ces petites molécules seraient ensuite absorbées dans l'hémolymphe par les parasites (Gordon et coll. 1971, Bailey et Gordon 1973, Gordon et coll. 1973, Condon et Gordon 1977, Rutherford et Webster 1978). Chez le Scolytide D. psendotsugae, Thong et Webster (1975) ont suggéré de la même manière une libération de tréhalose à partir du tissu adipeux, pour compenser les prélèvements de glucose par $C$. reversus dans l'hémolymphe. La modification du métabolisme des protéines et des sucres pourrait être causée par l'état de 
“ jeûne physiologique » dans lequel se trouvent les Insectes parasités (Gordon et coll. 1978, Schmidt et Platzer 1980).

Un autre mode d'action indirecte possible sur le tissu adipeux, et même sur les ovaires serait de nature endocrinienne. Dans ce cas les parasites pourraient agir sur le système neuroendocrinien de leur hôte, par l'intermédiaire de substances toxiques par exemple, comme l'ont suggéré Palm (1948) et Pouvreau (1962) chez Bombus sp. parasité par Sphaerularia bombi Dufour. Ils pourraient aussi émettre eux-mêmes des substances à activité hormonale, telles que l'analogue d'hormone juvénile sécrété par la microsporidie Nosema sp. chez les Insectes qu'elle parasite (Fisher et Sanborn 1964 in Gordon et coll., 1973).

Mise à part la possibilité d'une action enzymatique directe de $C$. diplogaster sur le tissu adipeux de son hôte, il n'est pas possible de choisir entre ces différentes hypothèses dans le cas qui nous préoccupe. Pour aller plus loin dans l'interprétation, il est nécessaire de procéder à une étude biochimique, au niveau de l'hémolymphe, du tissu adipeux et des ovaires d'I $p s$ sexdentatus.

Remerciements : L'auteur remercie C. Chararas, Directeur de recherches au CNRS (Institut National Agronomique, Paris), P. Pesson, Professeur honoraire à l'Institut National Agronomique, et C. Laumond, Maître de recherche à l'INRA (Station de Recherches sur les Nématodes, Antibes) d'avoir bien voulu relire et critiquer le manuscrit.

\section{BIBLIOGRAPHIE}

Ashraf M., Berryman A. A. : Biology of Sulphuretylenchus elongatus (Nematoda : Sphaerulariidae) and its effects on its host, Scolytus ventralis (Coleoptera : Scolytidae). Canad. Ent.,

I970a, 102, 197-213.
ASHRAF M., BERRYMAN A. A. : Histopathology of Scolytus ventralis (Coleoptera : Scolytidae) infected by Sulphuretylenchus elongatus (Nematoda: Sphaerulariidae). Ann. Ent. Soc. Am., 1970b, 64, 924-930.

BAILEY C. H., GoRdon R. : Histopathology of Aedes aegypti (Diptera : Culicidae) Larvae parasitized by Reesimermis nielseni (Nematoda: Mermithidae). $J$. Invert. Pathol., 1973, 22, 435$44 \mathrm{I}$.

Bovien P. : Some types of association between nematodes and insects. Vidensk. Medd. Dansk Naturh. Foren. Kobenhaven, 1937, I0I, II 4 p.

Chararas C. : Anatomie et biologie des Coléoptères Curculionides xylophages comparées à celles des Coléoptères Scolytides. Rev. Pathol. vég. entomol. France, 1956, 35 , II 3-214.

Chararas C. : Scolytides des Conifères, Lechevalier, Paris, 1962, 556 p.

Condon W. J., Gordon R. : Some effects of Mermithid Parasitism on the larval Blackflies Prosimulium mixtum fuscum and Simulium venustum. J. Invert. Pathol., 1977, 29, 56-62.

Fuchs G. : Die Naturgeschichte der Nematoden und einiger anderer Parasiten, I) des Ips typographus L., 2) des Hylobius abietis L. Zool. Jb., I91 5, 38, 109-222.

Gordon R., Condon W. J., Edgar W. J., BABIE S. J. : Effects of Mermithid parasitism on the hemolymph composition of the larval black-flies Prosimulium mixtum fuscum and Simulium venustum. Parasitology, 1978, 77, 367-374.

GORDON R., WEBSTER J. M. : Nutritional requirements for protein synthesis during parasitic development of the entomophilic nematode Mermis nigrescens. Parasitology, 1972, 64, I61172 .

Gordon R., WEBSter J. M., Hislop T. G. : Mermithid parasitism, protein turnover and vitellogenesis in the desert locust, Schistocerca gregaria Forskal. Comp. Biochem. Physiol., 1973, $46 B, 575-593$.

Gordon R., WebSTER J. M., MEAD D. E. : Some effects of the nematode Mermis nigrescens upon carbohydrate metabolism in the fat body of its host, the desert locust Schistocerca gregaria. Can. J. Zool., 1971, 49, 431-434. 
Gurando E. V. : Nematodes du petit Scolyte du Pin Blastophagus minor Hartig. Relations mutuelles avec leur hôte dans les régions boisées proches de Kiev. C. R. Thèse. Institut de Zoologie de l'A cademie des Sciences d'Ukraine, I974, 26 p. (en russe).

Hoffard W. M., Coster J. E. : Endoparasitic Nematodes of Ips Bark beetles in Eastern Texas. Environ. Entomol., 1976, 5, I28-132.

LAUMOND C., CARLE P. : Nematodes associés et parasites de Blastophagus destruens Woll. (col. Scolytidae) Entophaga, 1971, I6, 5I-66.

LIEUTIER F. : Le parasitisme d'Ips sexdentatus (Coleoptera, Scolytidae) par Contortylenchus diplogaster (Nematoda, Allantonematidae). Rev. Nématol., 1979, 2, 143-15I.

LIEUTIER F. : Le parasitisme d'Ips sexdentatus (BOERN). (Coleoptera, Scolytidae) par les Nématodes du genre Parasitaphelenchus Fuchs. Relations avec le parasitisme par Contortylenchus diplogaster (V. LrNs). Rev. Nematol., 1980, 3, 271-281.

LIEUTIER F. : Influence des nématodes parasites sur l'essaimage du Scolytide Ips sexdentatus (Boern.). Action régulatrice du froid. Acta Oecologica. Oecol. applic., 1981, 2, 357-368.

Lievtier F. : Action des nématodes endoparasites sur la ponte du Scolytide Ips sexdentatus Boern. (Insecta : Coleoptera). Acta Oecologica. Oecol., applic., 1982, 3, 204.

Lieutier F., Seureau C. : Encapsulement cellulaire de Contortylenchus diplogaster (V. LiNs.) (Nematoda : Allantonematidae) dans le tissu adipeux d'Ips sexdentatus (BOERN.) (Coleoptera : Scolytidae). Ann. Parasitol. Hum. Comp., 1981 56, 607-61 2.

MASSEy C. L. : The nematode parasites and associates of the fir engraver beetle, Scolytus ventralis Leconte, in New Mexico. J. Insect Pathol., 1964, 6, I33-I 55.

Nicholas W. L. : The fine structure of the cuticle of Heterotylenchus. Nematologica, 1972, 18 , I38I40.

NickLE W. R. : Observations on the effect of Nematodes on Ips confusus (Leconte) and other bark beetles. J. Insect. Pathol., I963, s, 386-389.

Oldham J. N. : On the infestation of Elm bark beetles (Scolytidae) by a Nematode, Parasitylenchus scolyti n. sp. J. Helminthol., I930, 8, 239-248.

Palm N. B. : Normal and Pathological histology of the ovaries in Bombus LAtr. (Hymenopt.) with hotes on the hormonal interrelations between the ovaries and the corpora allata. Opusc. Entomol. Suppl., 1948, VII, I-IOI.

Pornar G. O. : Nematodes as facultative parasite of Insects. Rev. Entomol., I972, 17, ro3-122.

PoINAR G. O. : Entomogenous Nematodes. A manual and host list of insect-nematodes associations, 317 p. E. J. Brill, Leiden, r975.

Pornar G. O., Hess R. : Romanomermis culicivorax : morphological evidence of transticular uptake. Exp. Parasitol., 1977, 42, 27-33.

Pouvreau A. : Contribution à l'étude de Sphaerularia bombi, parasite des reines de Bourdons. Ann. Abeille, 1962, s, I8I-199.

RIDING I. : Microvilli on the outside of a Nematode. Nature, 1970, 226, 179-180.

RuBtSov I. A. : Disposition et organes de la digestion extraintestinale des Mermithides. Izv. Akad. Nauk SSSR, Ser. Biol., I967, I0, 883-891. (en russe).

Ruнм W. : Die Nematoden der Ipiden. Parasit. Schriftenreihe, 1956, 6, 1-437.

RUtherford T. A., Webster J. M. : Some effects of Mermis nigrescens on the hemolymph of Schistocerca gregaria. Can. J. Zool., 1978, ,6, 339-347.

Rutherford T. A., Webster J. M., BARLow J. S. : Physiology and nutrient uptake by the entomophilic nematode Mermis nigrescens (Mermithidae). Can. J. Zool., r977, 55, I773-1781.

SAHOTA T. S. : Haemolymph and ovarial proteins in the bark beetle, Dendroctonus pseudotsugae in relation to ovarian development. Can. J. Zool., r970, 48, 1307-1312.

SAHOTA T. S., IBARAKI A. : Yolk deposition in the Douglas fir bark beetle, Dendroctonus pseudotsugae (Hopk.) : the significance of physiological state of oocytes. Can. J. Zool., I973, s1, 659$66 \mathrm{I}$.

Sahota T. S., Chapman J. A., Nijholt W. W. : Ovary development in a scolytid beetle Dendroctonus pseudotsugae (Ccleoptera : Scolytidae) : effect of farnesyl methyl ether. Canad. Ent., I970, $102,1424^{-1} 4^{28}$.

SAUNDERS J. L., NorRIS D. M. : Nematodes parasites and associates of the smaller European elm bark beetle, Scolytus multistriatus (Marsham). Ann. Entom. Soc. Am., 1961, 54, 792-798.

Schmidt S. P., Platzer E. G. : Changes in Body Tissues and Hemolymph Composition of Culex pipiens in Response to Infection by Romanomermis culicivorax. J. Invert. Pathol., 1980, 36, 240-254.

SCHVESTER D. : Contribution à l'étude écologique des Coléoptères Scolytides. Essai d'analyse des facteurs de fluctuation des populations chez Ruguloscolytus rugulosus MUller r9I8. Ann. I.N.R.A., Ser.C (Epiphyties), 1957, 8, 1-162.

Thong C. H. S., Webster J. M. : Effects of Contortylenchus reversus (Nematoda : Sphaerulariidae) on hemolymph composition and oocyte development in the Beetle Dendroctonus pseudotsugae (Coleoptera : Scolytidae). J. Invert. Pathol., 1975, 26, 91-98.

WACHEK F. : Die entoparasitischen Tylenchiden. Parasit. Schriftenreihe, I955, 3, I-I I9. 